\title{
Analysis, Design and Implementation of FSK Modulate Systems
}

\author{
Wu ChangFu
}

School of Mongolian Studies Inner Mongolia University,Hohhot 010021, China,imuwcf@126.com

Keywords: FSK Modulate System; FPGA; MATLAB

\begin{abstract}
The hybrid signals are the combined signals with multi-modulation mode. With multi-modulation, the disadvantages of single-modulation (such as FSK, PSK and PPM) can be eliminated. By taking the advantages of different modulations, the performance of range resolving, Doppler resolving and low probability of intercept (LPI) of the signal can be improved. FSK/PSK radar signal is the signal with both FSK and PSK modulation at one time. It is with large Bandwidth Time product (BT), thumbtack-like ambiguity function and well ability of Anti-EMI. The FSK/PSK signals of which the frequency-hopping and bi-phase sequences were randomly chosen often had high side lobe level. With the simulation and analysis using MATLAB, the method of FSK modulation is made analysis. As a result, the automatic modulation recognition is one of the necessary functions.
\end{abstract}

\section{Introduction}

With the development of the communication technology, the environment of wireless communications is becoming more and more complex. More and more modulation pattern with different modulation parameters are used in the wide frequency bands about the communication signals. How to effectively identify and monitor these signals, and it is the important research subject in the side of military and civilian[1]. Communication signal recognition and the parameter modulation pattern is the important content of COMINT. It is the premise to the communication interference and the protected reliably against detective. It can improve the automation degree of radio receiver and enhance the receiver real-time reconnaissance capability by the using of communication of automatic modulation signal recognition technology. In civilian areas, it can be used in signal interference proof, identification, frequency, management and so on. In recent years, the computer technology, digital signal processing technology and the rapid development of the special device makes the realization of automatic recognition about communications signal engineer become easy[2]. There are many good effects for the modulation technology. Firstly, change the base band signal into the modulated signal to fit in the channel of the signal transmission[3]; Secondly, improve the noise performance of the system; Thirdly, realize the channel multiplex. At present, more and more types of modulations are invented. And different modulation types will have different features and different modulation types will adapt to different places. Therefore, it is necessary to choose right modulation types in right place.

\section{The Analysis of FSK Modulation and Demodulation}

\section{The theory of FSK Modulation and Demodulation}

FSK modulation is one modulation mode to translate the information by changing the frequency of the carrier. It is one digital modulation technology that translates the information by using the base band digital signal discrete values to change the carrier frequency characteristics of the transfer information. And it is one of the earlier modulation modes. In FSK modulation, the frequency of the carrier is changed with the frequency change of base band signal and the frequency is changing between $f_{1}$ and $f_{2}$. Therefore, it can be decreased in the following equation.

$$
e_{2 F S K}(t)= \begin{cases}A \cos \left(w_{1} t+\varphi_{n}\right) & \text { when sending "1" } \\ A \cos \left(w_{2} t+\theta_{n}\right) & \text { when sending "0" }\end{cases}
$$


After analysis on the theory of the FSK modulation, the signal after FSK modulation can be seen as the sum of the two frequencies signals. Therefore, we can change the FSK modulation signal into two signals with different frequencies. And the equation about FSK modulation will be changed.

$$
e_{2 F S K}(t)=\left[\sum_{n} a_{n} g\left(t-n T_{s}\right)\right] \cos \left(w_{1} t+\varphi_{n}\right)+\left[\sum_{n} \overline{a_{n}} g\left(t-n T_{s}\right)\right] \cos \left(w_{2} t+\theta_{n}\right)
$$

In the equation, $g(t)$ is one rectangular pulse, and the width of the signal is $T_{s}$.

$$
a_{n}= \begin{cases}1 & \text { the probability is } P \\ 0 & \text { the probability is } 1-P\end{cases}
$$

The code of signal named $\overline{a_{n}}$ is contrary to the signal named $a_{n}$. When the value of $a_{n}$ is 0 , the value of $\overline{a_{n}}$ is 1 . In the other side, when the value of $a_{n}$ is 1 , the value of $\overline{a_{n}}$ is 0 . Therefore, the probability of $\overline{a_{n}}$ can be shown in the following equation.

$$
\overline{a_{n}}= \begin{cases}1 & \text { the probability is } 1-P \\ 0 & \text { the probability is } P\end{cases}
$$

It is easy to see that the value of $\varphi_{n}$ and $\theta_{n}$ is only show the initial phase of the code numbered $n$. but the information is not only translated by $\varphi_{n}$ and $\theta_{n}$. As usual, the value of $\varphi_{n}$ and $\theta_{n}$ can be set as 0 . Therefore, the formula can be change as following.

$$
e_{2 F S K}(t)=s_{1}(t) \cos w_{1} t+s_{2}(t) \cos w_{2} t
$$

In this equation, signal about $s_{1}(t)$ and $s_{2}(t)$ can be described as following.

$$
\begin{aligned}
& s_{1}(t)=\sum_{n} a_{n} g\left(t-n T_{s}\right) \\
& s_{2}(t)=\sum_{n} \overline{a_{n}} g\left(t-n T_{s}\right)
\end{aligned}
$$

The theory of FSK modulation and demodulation will be shown in the following figures with the help of software named MATLB[3].

As shown in figure1 and figure2, the analysis of theory of FSK can be more directly. In figure2, we can get the bandwidth of signal after FSK can be shown in the following equation.

$$
B_{F S K} \approx\left|f_{2}-f_{1}\right|+2 f_{s}
$$
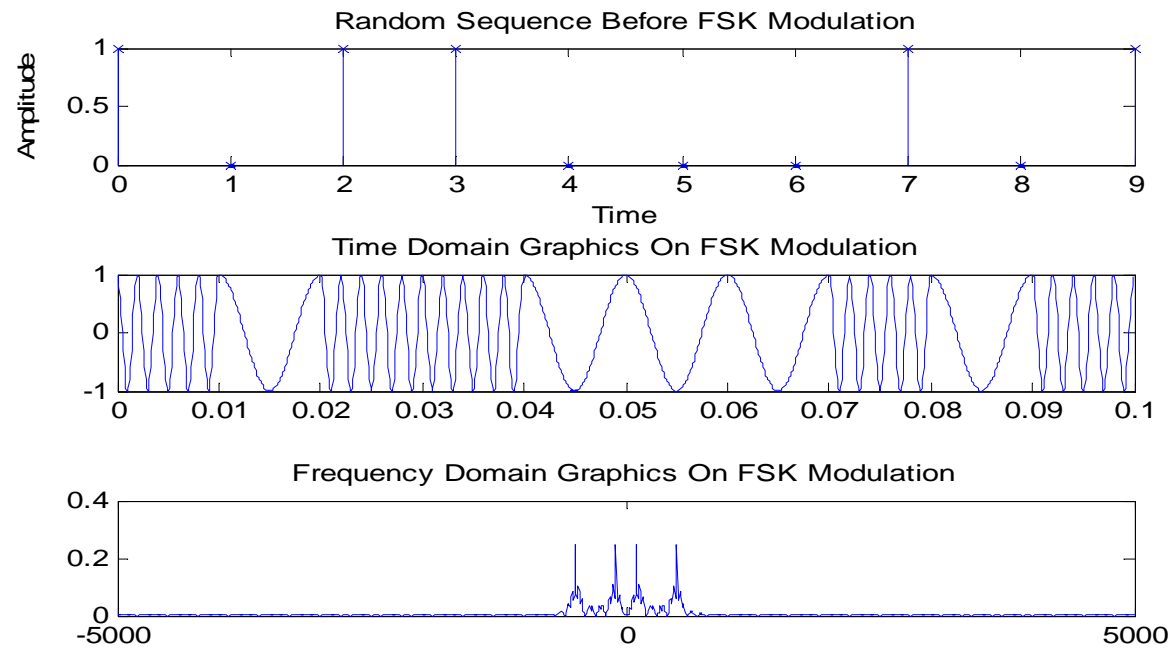

Figure.1 Theory of FSK Modulation and Demodulation 

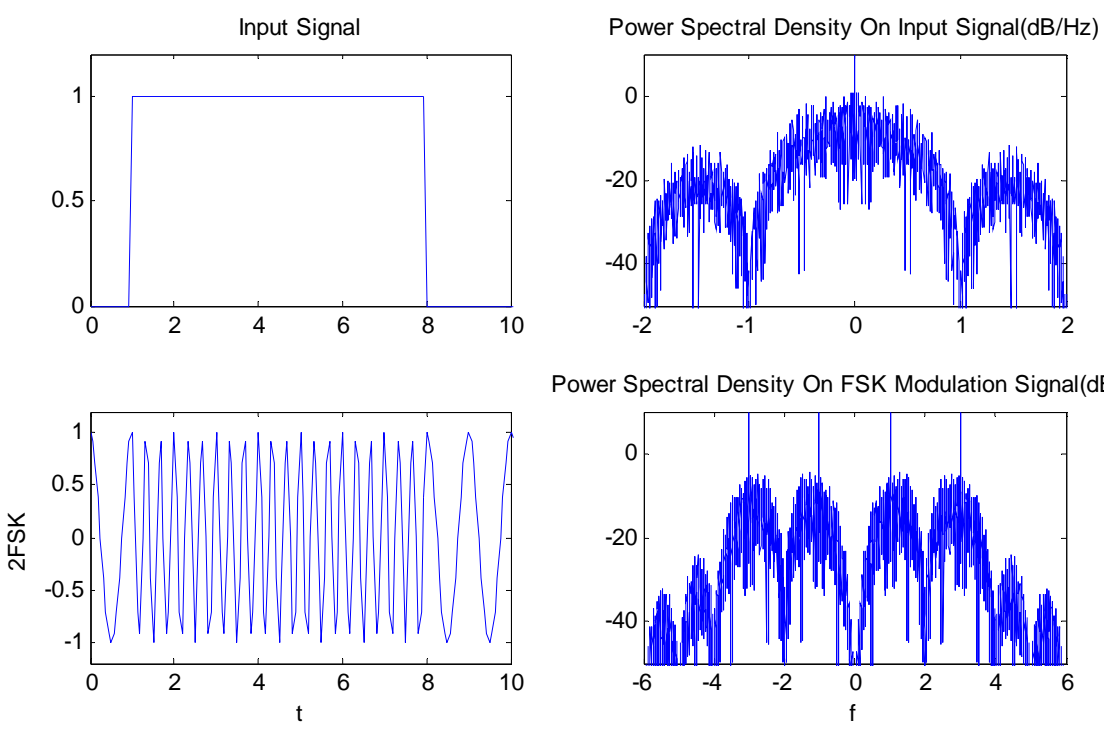

Power Spectral Density On FSK Modulation Signal $(\mathrm{dB} / \mathrm{Hz})$

Figure.2 Analysis on Power Density of FSK Signal

\section{The Realization of FSK modulated System based on FPGA}

\section{Introduce}

FPGA is the short of Field programmable gates array. And it is a new type of PLD, the first production manufacturers is named Xilinx in America in 1985. It becomes more and more famous even when it is invented. The device has become a good choice for the electronic design engineer because of the feature of large scale, high flexibility, more widely realization of logic function [4-5]. The speed requirement of circuit becomes higher and higher, with the development of the computer science and the communication technology. The traditional circuit can not meet the requirement of the high efficiency and real-time processing, and it need time to produce new type devices. But FPGA can be a good choice, it can meet the requirement of the design, and it can be reusable, the confidentiality of FPGA design is better than the other devices. We can use hardware description language (HDL) to design the circuit in FPGA. In this paper, the realization of FPGA modulated system will be based on the FPGA.

The Realization of FSK Modulation system based on FPGA

The realization of FSK modulation and demodulation is mainly based on FPGA and the hardware description language (HDL). For FPGA design, we must abide by its design method. Firstly, described the logic circuit of FSK modulation and demodulation based on the above analysis, and make sure the hardware description language is right. Secondly, make simulation based on the right HDL, and make sure the result of simulation is right. Thirdly, download the bit stream to FPGA device. if the result of simulation is wrong, the hardware description language will be changed until the result of simulation is right. Fourthly, download the bit stream to FPGA device and see the result by the software or oscilloscope. The simulation results and the fact results will be described in the following.

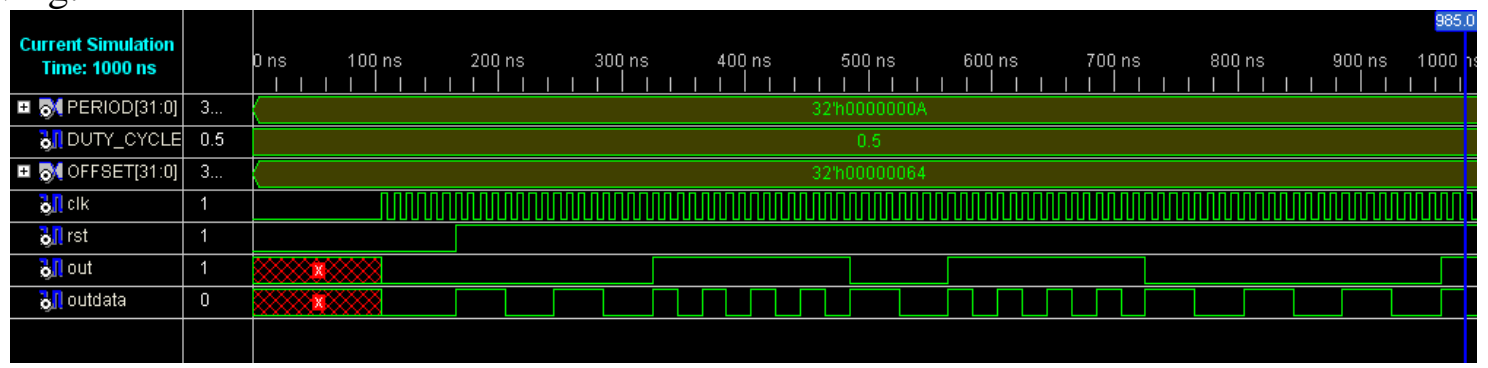

(a) 


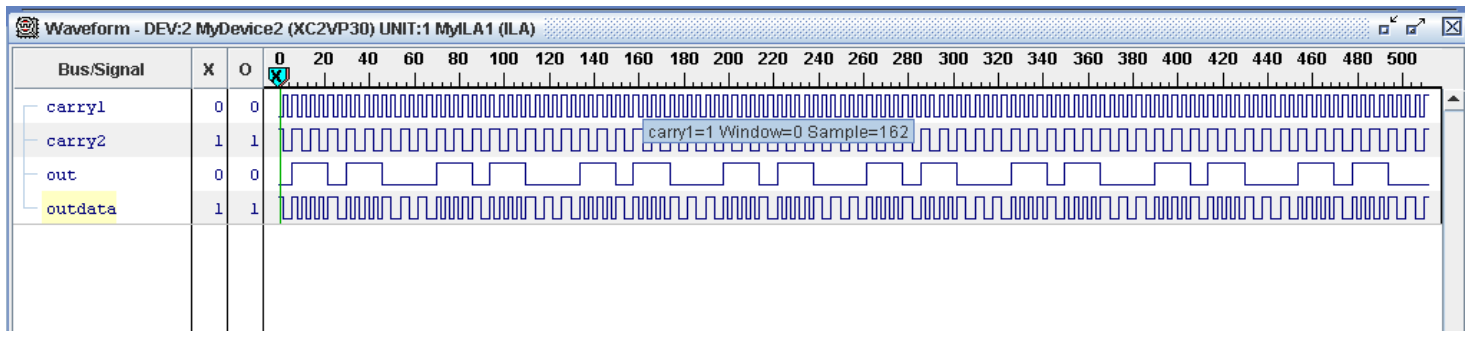

(b)

Figure.3 Result of Simulation and on FPGA

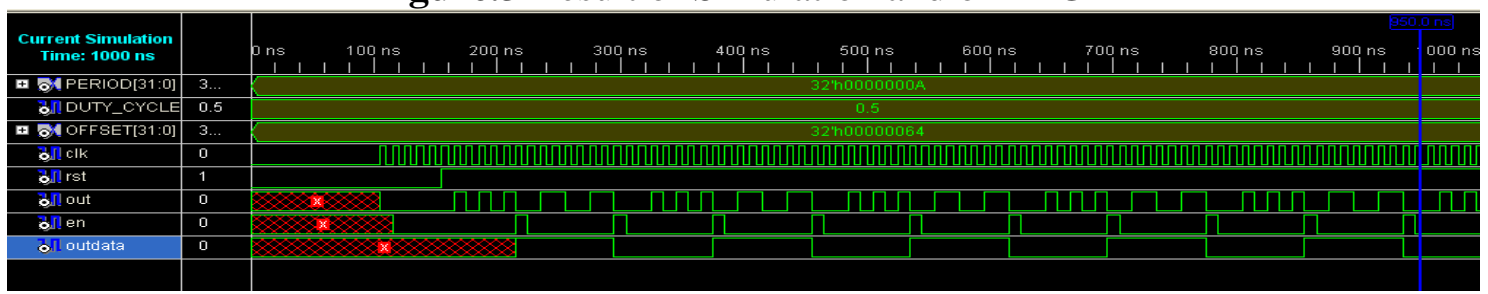

(a)

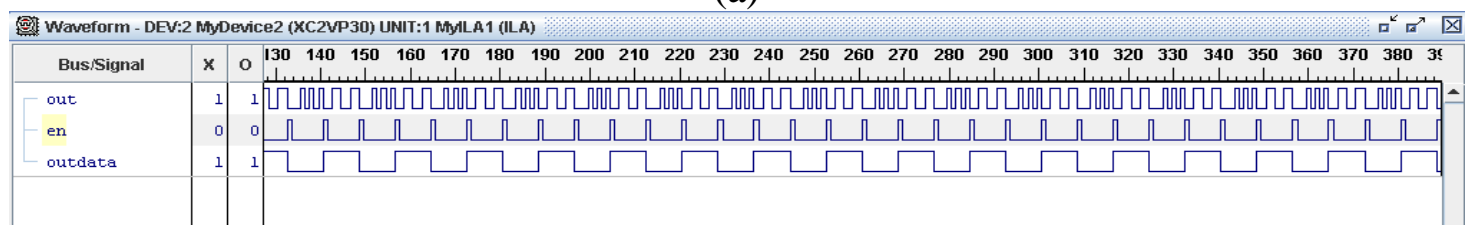

(b)

Figure.4 Result of Simulatin and on FPGA

Picture (a) in figure3 and picture (a) in figure4 are the simulation results of FSK modulation and demodulation. Picture (b) in figure3 and picture (b) in figure4 are the fact results on FPGA of FSK modulation and demodulation. From the results above, we can know that the realization of FSK modulation system based on FPGA is well, and the fact results can be accepted.

\section{Summary}

In this paper, author makes some analysis on the method of FSK modulation and demodulation, and some widely useful methods are also described. with the help of MATLAB, the features of FSK modulation can be saw easily. In this paper, the realization of the FSK modulation system is based on FPGA, and the results are also shown in figure7 and figure8, compared with other methods, I make sure this method can be accepted. I believe with the development of the communication, more and more methods will be invented, but I think the modulation system based on FPGA will be a good choice.

\section{References}

[1] Raytheon Co., Wayland, Mass.: The Performance of FSK Permutation Modulations in Fading Channels and Their Comparison Based on A General Method for the Comparison of M-ary Modulations, Communication technology, Vol. p.616-640.

[2]Fujita, T, Kawanishi, T:OOK/FSK WDM System Using External Optical FSK Modulation, Optical Communication, Vo3. p.785-786.

[3] Luhe Hong, Jianli Cai: The Application Guide of Mixed Programming Between MATLAB and Other Programming Language, Computer and Automation Engineering, Vo3. p.185-189.

[4] Mayer-Lindenberg, F: High-Level FPGA Programming through Mapping Process Networks to FPGA Resources, Reconfigurable Computing and FPGAs, Vo2. p.302-307.

[5] Banatre, J.P. et.al,From High Level Programming Model to Fpga Machines, IEEE Workshop on FPGAs for Custom Computing Machines,pp.10-13,1994 